\title{
Control of Laminar Separation Bubble over a NACA2415 Aerofoil at Low Re Transitional Flow Using Blowing/Suction
}

\author{
M. Serdar GENÇ*, Ünver KAYNAK ${ }^{* *}$
}

\begin{abstract}
In this study, control of flow over a NACA2415 aerofoil which experiences a laminar separation bubble at a transitional Reynolds number of $2 \times 10^{5}$ is computationally investigated using blowing or suction. In an earlier flow control study which involved the use of a leading edge slat, both the experimental and computational studies demonstrated significantly delayed stall angles. For these flows, employing the recently developed $k-k_{L}-\omega$ and k- $\omega$ SST transition models were shown to accurately predict the location of the experimentally determined separation bubble. In the present case, a single jet with a width of $2.5 \%$ the chord length is placed on the aerofoil's upper surface simulating the blowing/suction control at $\mathrm{Re}=2 \times 10^{5}$ and $\alpha=8$ degrees. Nearly 200 numerical simulations are carried out a range of parameters of jet locations, $L_{j e t}$, jet velocity ratios, $R_{\text {jet }}$ and jet angles, $\theta_{\text {jet }}$ are investigated. In the numerical results of blowing/suction, it is shown that the smaller blowing results are better larger blowing velocity ratios while the largest suction results are better smaller suction velocity ratios independent of the suction jet locations.
\end{abstract}

Keywords: Laminar separation bubble, Flow separation control, Blowing, Suction, Transition models.

\footnotetext{
* Student, School of Civil Aviation, University of Erciyes, Kayseri, 38039, Turkey, E-mail: musgenc@erciyes.edu.tr, (Corresponding author).

** Professor, Department of Mechanical Engineering, TOBB University of Economics and Technology, Ankara, 06560, Turkey, E-mail: ukaynak@etu.edu.tr
} 


\section{Nomenclature}

$\begin{array}{ll}\alpha & =\text { angle of attack of the main aerofoil } \\ \alpha_{s} & =\text { angle of attack at which the main aerofoil stalls } \\ c & =\text { aerofoil chord length } \\ C_{f} & =\text { skin friction coefficient } \\ C_{P} & =\text { pressure coefficient } \\ C_{L} & =\text { lift coefficient } \\ C_{\text {Lmax }} & =\text { maximum lift coefficient } \\ \varepsilon & =\text { turbulent dissipation } \\ \mathrm{k} & =\text { turbulent kinetic energy } \\ \mathrm{k}_{\mathrm{T}} & =\text { turbulent kinetic energy } \\ \mathrm{k}_{\mathrm{L}} & =\text { laminar kinetic energy } \\ \mu & =\text { moleculer viscosity } \\ \mu_{\mathrm{T}} & =\text { eddy viscosity } \\ \mathrm{NACA} & =\text { National Advisory Committee for Aeronautics } \\ p & =\text { pressure } \\ \mathrm{Re} & =\text { Reynolds number } \\ \mathrm{Re}_{\theta \mathrm{t}} & =\text { transition onset momentum thickness Reynolds number } \\ V_{\infty} & =\text { free stream velocity } \\ x & =\text { axis parallel to the chord line } \\ \omega & =\text { specific turbulence dissipation rate } \\ T u & =\text { turbulence intensity, } \% \\ \mathrm{y}^{+} & =\text {normal distance in wall coordinates } \\ z & =\text { axis normal to the chord line } \\ \gamma & =\text { intermittency } \\ \theta_{\text {jet }} & =\text { Jet angle } \\ \mathrm{L}_{\text {jet }} & =\text { Jet location }(\% \mathrm{c}) \\ \mathrm{V}_{\text {jet }} & =\text { Jet velocity (m/s) } \\ & \end{array}$

\section{Introduction}

Typical air speeds at high angles of attack for micro-air vehicles and Unmanned Air Vehicles (UAVs) involve transitional Reynolds number (Re) regime, where laminar separation bubbles appear on the suction surface of the wings. Delaying, eliminating or controlling such separation bubbles are important aircraft design issues, and measuring, modeling and predicting them are challenging research problems.

The objective of the flow control is to manipulate a particular flow field with a small energy input typically aiming to increase the lift and reduce the drag, to enhance the mixture of momentum, energy, and species, and to suppress the flow-induced noise. Examples of techniques to obtain these outcomes are: delay or advance transition, prevent or provoke separation, and suppress or enhance turbulence. The addition of suction or blowing controls in experiments will paradoxically require finer measurements of sensitive and smaller scale flows. Yet, they will increase the complexity of the overall flow accompanied with a further rise in the experimental error. Trying to repeat these experiments over a wide range of 
potential parameters necessary to determine the optimal performance conditions for an active flow control design would necessarily be expensive. The alternate approach is to use numerical simulation for more affordable, practical, and systematic solution.

In parallel with modern developments in experimentally capturing, measuring, and identifying the laminar separation bubbles that are typical in the low-speed flow regimes, improved prediction methods to account for transition mechanisms over wings have been devised. High performance computing capabilities make it possible to routinely use RANS based CFD methods for simulating transitional and turbulent flows. Today, state of the art RANS solvers frequently include practical one- or two-equation turbulence closure models [15].

Transition predictions have also progressed by means of the $\mathrm{e}^{\mathrm{N}}$ method [9], two-equation low Re-number turbulence models [13], and early methods based on experimental correlations [8]. The $\mathrm{e}^{\mathrm{N}}$ method has been quite successful in practice and more or less has become the industry standard. Standard low-Re models have also shown success, though the wall damping terms' ability to capture important transition effects limits their use. Some early correlation-based models have become helpful tools for industry, although they have limitations due to their use of integral (or global) boundary layer parameters and thus not amenable to practical use in the RANS based methods.

Recently, transport equation models $[22,25]$ which rely on "local" data to circumvent some complicated procedures in the early methods, have been introduced. These methods solve several "transport" partial differential equations written for different transition quantities in addition to the baseline turbulence models. These models have been made available [24] in some commercial CFD codes. However, in the so-called "engineering transition model" [22] the correlations either remain proprietary [24], or user-dependent [26] based on the available experimental data. In order to assess user-dependent transition correlations, Misaka and Obayashi [26] and Kaynak and Gurdamar [29] published some transition formulae.

Control of flow separation and transition point by means of different mechanisms such as using leading edge devices [14], blowing, and suction have been quite extensively researched. Wu et al. [16] investigated control effects on a NACA 0012 airfoil with a local unsteady forcing $(2.5 \%$ chord length width) located at $5 \%$ from the leading edge at the angle of attack from $18^{\circ}$ to $35^{\circ}$ with a 2-D RANS approach. Catalin [17] studied control effects on a NACA 0012 airfoil with synthetic jet array (10\% width) located at $10 \%$ from the leading edge at the angle of attack of $13^{\circ}$. Hassan et al. [18] studied a synthetic jet located at $13 \%$ from the leading edge at the angle of attack of $0^{\circ}$ and $5^{\circ}$. All of the above studies find that the synthetic jet and forcing/non-forcing (oscillatory/steady) suction/blowing on the aerofoil leading edge can increase lift and decrease drag. Shan et al. [30] studied subsonic flow separation over a NACA0012 airfoil with a $6^{\circ}$ angle of attack and flow separation control with vortex generators. They investigated three cases including an uncontrolled baseline case, a controlled case with passive vortex generator, and a controlled case with active vortex generator. The size of the averaged separation zone has been reduced by more than $80 \%$. It was shown that the flow control with active vortex generator was more effective and the separation zone was not visible in the averaged results. Huang et al. [23] presented the blowing and suction control on a NACA0012 airfoil under $\mathrm{Re}=5 \times 10^{5}$ at angle of attack $18^{\circ}$.

In this study, control of flow over NACA2415 aerofoil is computationally investigated using blowing/suction. A single jet with a width of $2.5 \%$ the chord length is placed on the aerofoil's upper surface simulating the blowing/suction control under $\mathrm{Re}=2 \times 10^{5}$ at the angle of attack of $8^{\circ}$. The jet width for blowing/suction is fixed at $2.5 \%$ chord length based on a study by 
Dannenberg and Weiberg [1] who showed that an increase of suction area beyond $2.5 \%$ chord length will not increase lift significantly. The critical values of blowing/suction locations (three locations; on 10\%-before bubble, 26\%- center of bubble, 36\%-after bubble-the chord length), jet velocity ratios $(0.003,0.016,0.032,0.064,0.156)$, and angles $\left(30^{\circ}, 45^{\circ}, 90^{\circ}\right)$ are discussed. Note that negative $\theta_{j e t}$ represents suction condition and positive $\theta_{j e t}$ indicates blowing condition.

\section{Numerical Method}

\section{Flow solvers}

Calculations were performed over the NACA 2415 aerofoil with blowing to study the laminar separation bubble and transition location. In an earlier work [32-35], baseline calculations using the single- and multi-element aerofoil configurations provide confidence in the CFD solvers by comparing the numerical results with experimental data. The freely-available prediction code, XFOIL [15] and the commercial code, FLUENT were used as a baseline codes.

The XFOIL code is a viscous-inviscid boundary layer interaction code which employs the industry standard $\mathrm{e}^{\mathrm{N}}$ method to model transition. The FLUENT code solves the Reynolds averaged Navier-Stokes equations using finite volume discretization. Second order upwind discretization in space is used, and the resulting system of equations is then solved using the SIMPLE coupled solution procedure until convergence criteria are satisfied. The convergence rate is monitored during the iteration process by means of the residuals of the dependent variables of the governing differential equations. A convergence criteria of $\mathrm{O}(5)$ reduction in all dependent variable residuals is accepted as adequate for the present aerofoil study. Convergence is also checked using the relative differences between two successive iterations for each of the integrated force and moment coefficients. A free stream turbulence level of $\mathrm{Tu}$ $=0.1 \%$ is used and the ratio of the turbulent to molecular viscosities is set to $\mu_{\mathrm{T}} / \mu_{\mathrm{L}}=1$ in the FLUENT calculations. Free stream boundary conditions are used in the upstream, downstream and outer boundaries. No-slip boundary conditions are used at solid surfaces.

\section{Transition models}

Laminar-turbulent transition plays a major role in low Reynolds number aerofoil predictions, including the formation of the separation bubble and its effect on the stall phenomenon. Until recently industry generally relied upon either low Re turbulence models [13], or experimental correlations $[8,19,20]$ which usually relate the turbulence intensity to the momentum thickness Reynolds number at transition onset. Early experimental correlation methods were commonly implemented within boundary layer type solvers as they lend themselves to global, or integral parameters in the boundary layer. The recent introduction of "engineering transition models", or the "transport equation models", have helped overcome certain restrictions emanating from the use of global parameters within RANS type solvers. Two such models were the correlation-based $k-\omega S S T$ Transition Model of Menter et al. [22] and the $k$ $k_{L^{-}} \omega$ transition model of Walters and Leylek [25]. Both models are suitable for straightforward implementatation within RANS methods as they solve additional transport equations for predicting transition phenomena that rely on local information only, in contrast with the global information, as used in the early methods. 
The $k-\omega S S T$ transition model [22] is based on two transport equations: the first is an intermittency equation ( $\gamma$-equation), used to trigger the transition process; and the second is the transition onset momentum thickness Reynolds number ( $\operatorname{Re}_{\theta t}$-equation) which is forced to follow experimentally-determined correlations. Correlations are user dependent, based upon the best experience of from individual laboratories, and various authors have tried to develop sensible correlations for different experimental cases [26, 29]. In this model, the Shear Stress Transport (SST) feature is linked to the transition model by coupling it with the $k-\omega S S T$ turbulence model [28]. This is essentially a hybrid turbulence model that couples the standard $k-\varepsilon$ and $k$ - $\omega$ models in an efficient manner in which the $k$ - $\omega$ model (in the near-wall region) and the standard $k-\varepsilon$ model (in the far-field region) are blended together, including the modeling of transport of shear stress via modified definition of the turbulent viscosity.

The $k-k_{L^{-}} \omega$ model [25] is considered as a three-equation eddy-viscosity type, which includes transport equations for turbulent kinetic energy $\left(k_{T}\right)$, laminar kinetic energy $\left(k_{L}\right)$, and specific dissipation rate $(\omega)$. Presently, both methods are used selectively in the literature and are shown to produce successful results. In a recent paper, Cutrone et al. [28] have evaluated both the early correlation based models $[8,19]$ and the $k-k_{L^{-}} \omega$ model [25] for a number of twodimensional incompressible separated flows past a flat plate with semi-circular leading edge, and a three-dimensional T106 linear turbine cascade flow. Suluksna and Juntasaro [31] assessed the performance of the early correlation methods [23] and the transport equation based RANS methods [21, 22] against the European Research Community on Flow, Turbulence and Combustion Database (ERCOFTAC) [12] flat plate experimental test cases.

\section{Blowing/Suction}

In the study of the single blowing and suction jet control computationally investigated because the experimental study of the blowing/suction was expensive and difficult and three parameters are used in the investigation as shown in Fig. 1. The parameters are the jet location, $\mathrm{L}_{\text {jet }}$, jet speed, $\mathrm{V}_{\text {jet}}$, and the blowing/suction angle, $\theta_{\text {jet. }} \theta_{\text {jet }}$ is the angle between the local jet surface and jet entrance velocity direction. Note that negative $\theta$ represents suction condition and positive $\theta$ indicates blowing condition. A single jet with a width of $2.5 \%$ the chord length is placed on the aerofoil's upper surface simulating the blowing/suction to control of laminar separation bubble under $\mathrm{Re}=2 \times 10^{5}$ at 8 degrees. The jet width for blowing/suction is fixed at $2.5 \%$ chord length based on a study by Dannenberg and Weiberg [1] who showed that an increase of suction area beyond $2.5 \%$ chord length will not increase lift significantly. The critical values of blowing/suction locations (three locations, $\mathrm{L}_{\mathrm{jet}}$; on $10 \%$-before bubble, $26 \%$ - center of bubble, $36 \%$-after bubble-the chord length), $\mathrm{R}_{\text {jet }}$ velocity ratios $(0.003,0.016,0.032,0.064,0.156)$, and jet angles, $\theta_{\text {jet }}\left(30^{\circ}, 45^{\circ}, 90^{\circ}\right)$ are discussed. Jet entrance velocity is defined as

$$
\begin{aligned}
& u=R_{j e t} \cos \left(\theta_{j e t}+\beta\right) \\
& v=R_{j e t} \sin \left(\theta_{j e t}+\beta\right)
\end{aligned}
$$

where $\beta$ is the angle between the freestream velocity direction and the local jet surface, $\theta_{\text {jet }}$ is the angle between the local jt surface and jet entrance velocity direction. 


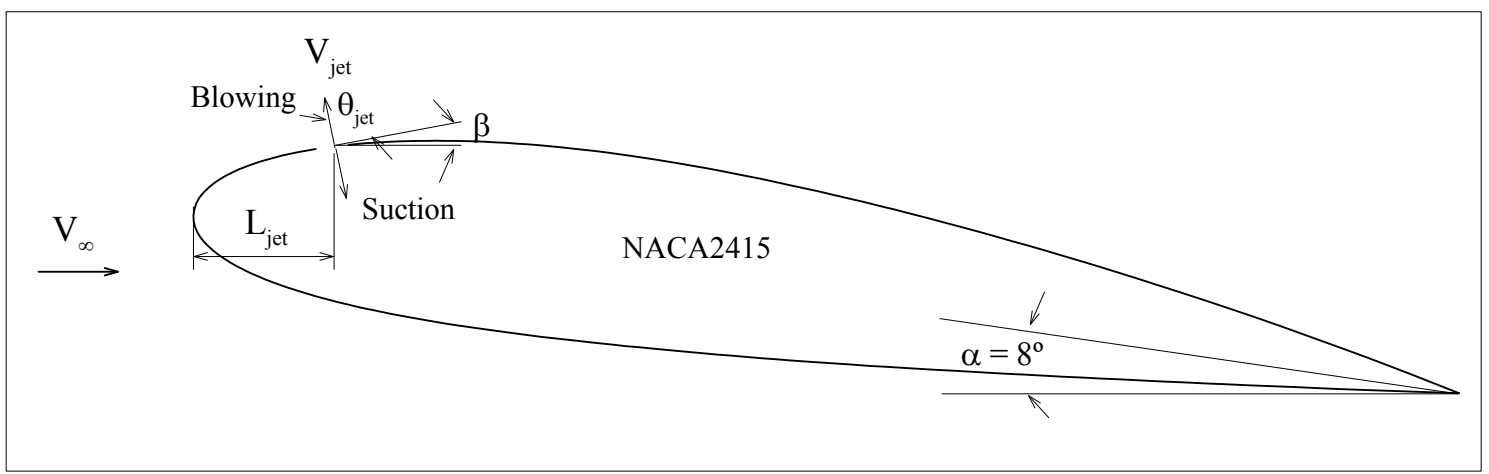

Figure 1. Blowing control mechanism.

\section{Solution Grid}

The grid used for the single aerofoil is generated by the GAMBIT program, and is shown in Figure 2. The grid extends from -10 chords upstream to 20 chords downstream. The upper and lower boundary extends 10 chords from the profile. The grid shown is a " $\mathrm{C}$ " like structured grid. Different size grids are used to ensure grid independence of the calculated results. This is achieved by obtaining solutions with increasing number of grid nodes until a stage is reached where the solution exhibits negligible change with further increase in the number of nodes. Consequently, the grid size giving the grid independent results are selected and the total number of cells is adopted as 35,000 nodes.

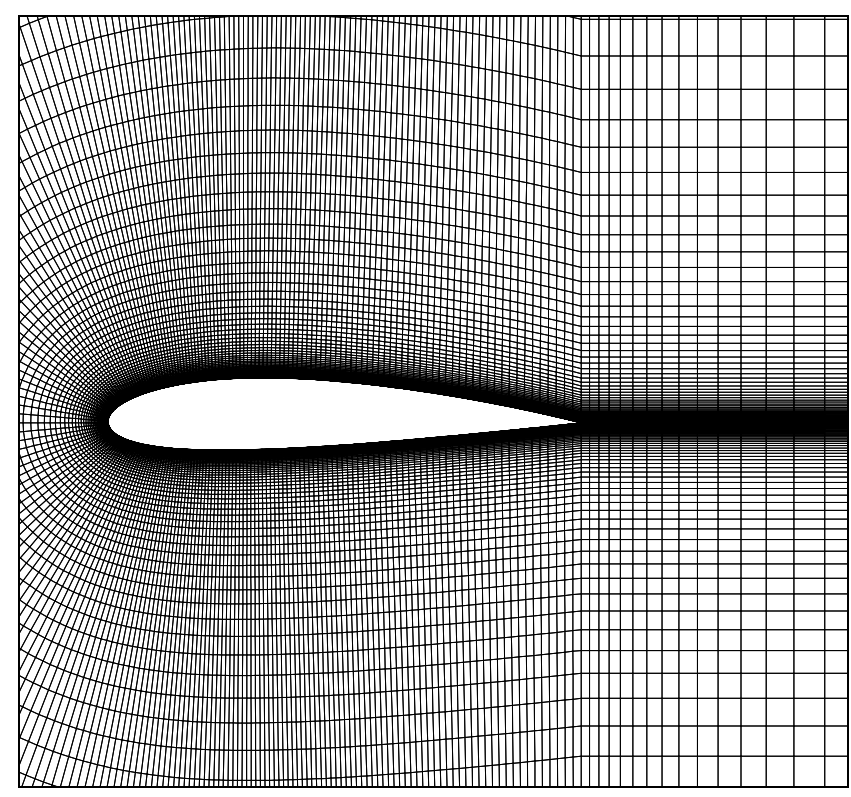

Figure 2. Structured grid of single NACA 2415 aerofoil for CFD analysis. 


\section{Results and Discussions}

\section{A. Numerical and Experimental Results of Single NACA 2415 Aerofoil}

In order to validate the wind tunnel set-up in the low Reynolds number range, the NACA 2415 aerofoil was first tested by running the wind tunnel at $20 \mathrm{~m} / \mathrm{s}$, which gave a Reynolds number in the range of $2 \times 10^{5}$. The angle of attack of the NACA 2415 aerofoil was adjusted in one degree intervals and the lift and drag moment coefficients. Figure 3 shows the lift coefficient $\left(C_{L}\right)$ and drag coefficient $\left(C_{D}\right)$ versus angle of attack $(\alpha)$ curve from the wind tunnel experiments and compares them with the numerical data calculated from the XFOIL and FLUENT codes at Reynolds numbers of $2 \times 10^{5}$. The maximum lift coefficient defines the angle at which the aerofoil will stall and this is shown to vary with the wind tunnel. In Bath University [32,33], the stall occurred at the angle of attack of $12^{\circ}$ where $C_{\text {Lmax }}=1.33$ whereas in TOBB ETU [34-36] at the angle of attack of $14^{\circ}$ where $C_{\text {Lmax }}=1.35$. However, the XFOIL solution reflects the viscous-inviscid interaction approach with the $e^{N}$ transition method and is in good agreement with the experimental data in the linear region. The FLUENT solutions, which include the results of the low Reynolds number turbulence model, full turbulence models and transition models, are also shown. Firstly, the $k-\varepsilon$ RNG model and the low Reynolds $k$ - $\omega$ models were used as the baseline turbulence models to predict the performance of low Re and fully turbulent approach without transition. Secondly, the $k-\omega$ SST transition and the $k-k_{L}-\omega$ transition models were used to predict the transition effects under the transport model assumption. In Figure 3, all numerical approaches give reasonably good results against the experiment in the linear region, whereas discrepancy is noted as stall is approached. There are mixed results from the different models: the low Re number $k-\omega$ and $k-\omega$ SST transition models underpredict, and the fully turbulent $k-\varepsilon$ RNG overpredict the stall. But, $k-k_{L^{-}} \omega$ transition model and the $e^{N}$-XFOIL are in good agreement with the experimental data. In $k-k_{L^{-}}$ $\omega$ transition model, the stall occurred at the angle of attack of $14^{\circ}$ where $C_{L \max }=1.38$ whereas in $e^{N}$-XFOIL at the angle of attack of $14^{\circ}$ where $C_{\text {Lmax }}=1.29$. Overall, the $k-k_{L}-\omega$ model seems to be better than other transition models for predicting the lift.

The graph depicted in Figure 3 also shows the drag coefficient variations with the angle of attack. There is quite good agreement between the experiment and computations for low angles of attack. However, the experimental $C_{D}$ values are somewhat less than the numerical $C_{D}$ values at low angles of attack. Here, the small influence of the shear stress was not taken into account in the experimental $C_{D}$. This is due to certain limitations which made it impossible to establish the skin friction drag and consequently all the drag coefficient results are pressure drag coefficients in the experiment.

Figure 4 shows the numerical and experimental using hot-wire anemometer in TOBB ETU velocity profiles over the upper surface of the NACA 2415 aerofoil at the angle of attack of $8^{\circ}$. Individual figures clearly show the separation bubble and the adverse velocity profiles at various chordwise positions. Both transition models and the $k-\omega$ SST turbulence model predict more pronounced reverse flow regions, but the $k-\varepsilon$ RNG turbulence model misses the separation bubble. As shown, the wall velocity gradient and the wall shear stress are zero at start of the separation $(15 \% \mathrm{c}$ ). Beyond that location (the profiles from $20 \% \mathrm{c}$ to $30 \% \mathrm{c}$ ), there is reverse flow in the boundary layer. Transition from laminar to turbulent flow within the bubble also involves a noticeable change in the shape of the boundary layer velocity profile. As shown in the $35 \%$ station, turbulent profiles are flatter, have larger velocity gradients at the wall, and produce larger boundary layer thickness than do the laminar profiles $(10 \% \mathrm{c})$. 
It appears that the $k-k_{L}-\omega$ transition model better depicts the transition from laminar to turbulent flow with more pronounced velocity profiles and changing thickness of the separation bubble within the boundary layer.

Oil flow visualization is a relatively simple way of examining surface flow patterns. In order to display the separation bubble, the upper surface oil flow pattern for the NACA 2415 aerofoil at $8^{\circ}$ are shown in Figure 9. The oil flow experiment has identified a laminar separation bubble on the suction surface of the NACA 2415 around $30 \%$ chord.

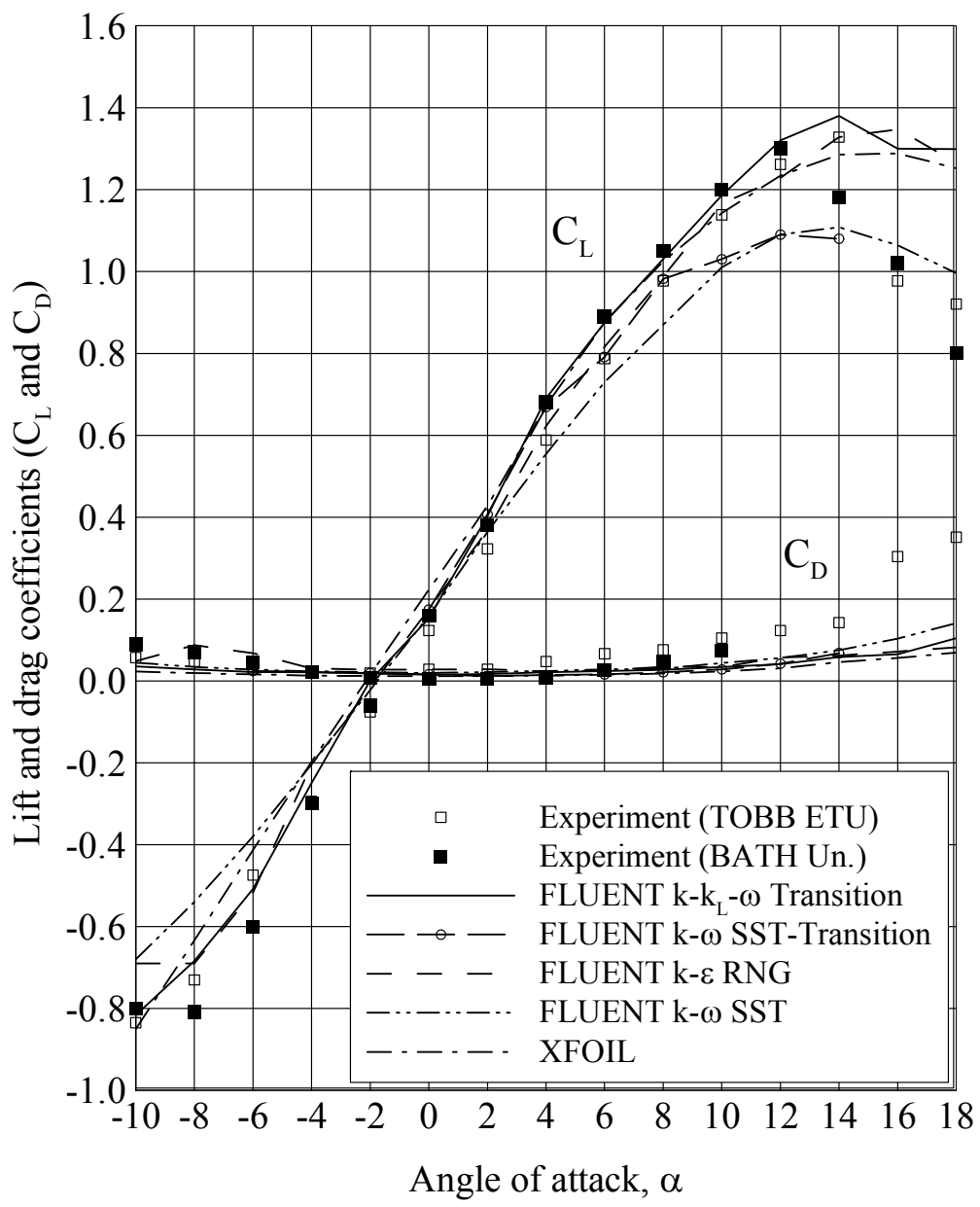

Figure 3. Lift and drag coefficients of the NACA 2415 aerofoil at different angles of attack. 


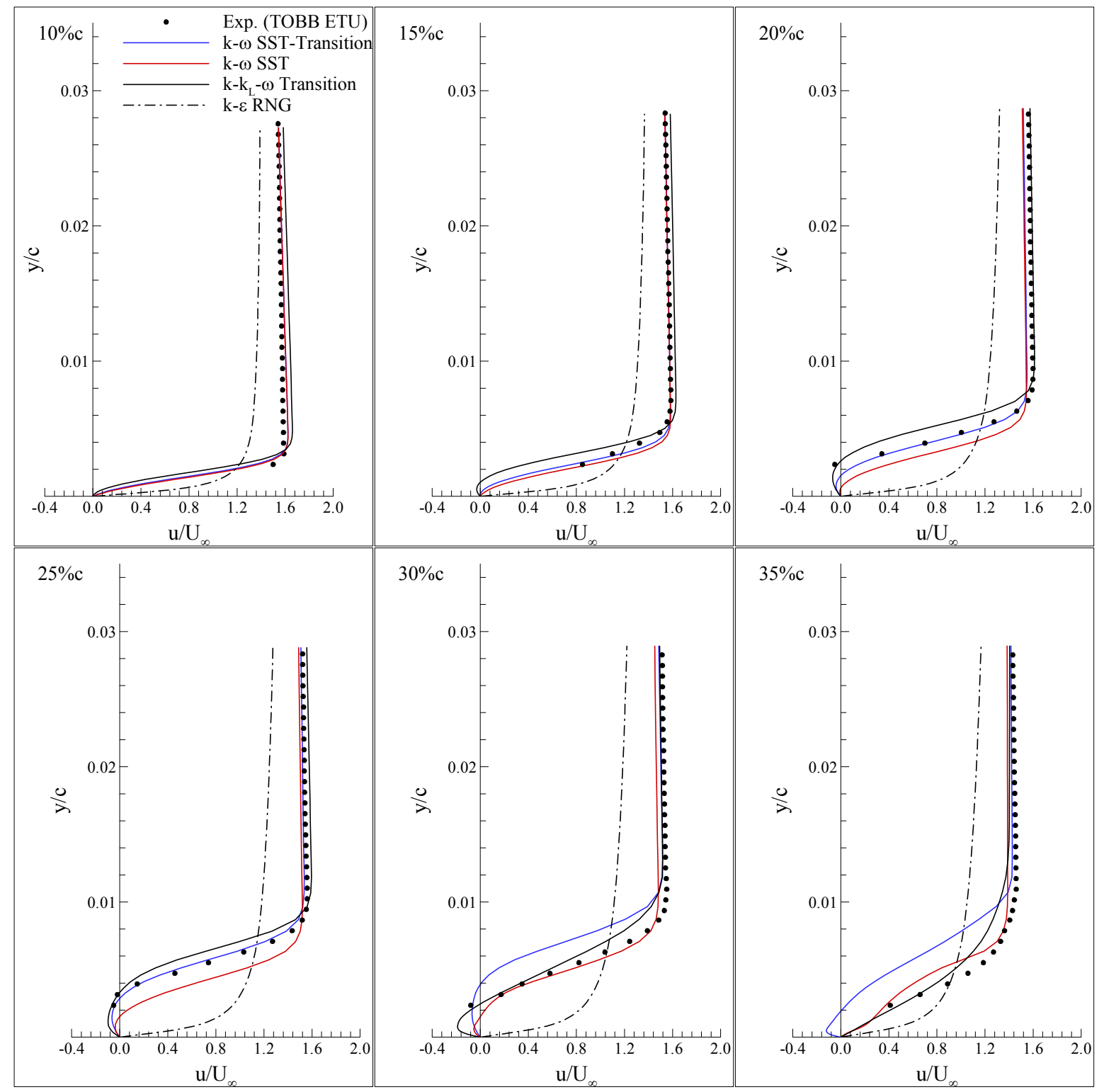

Figure 4. Numerical and experimental using hot-wire anemometer in TOBB ETU velocity profiles over the upper surface of the NACA 2415 aerofoil at an angle of attack of $8^{\circ}$. 


\section{B. Numerical Results of the NACA 2415 Aerofoil using Blowing/Suction}

Figure 6 shows the pressure distributions over the single element NACA 2415 aerofoil at $\alpha=8^{\circ}$ without and with the blowing/suction. In an earlier experimental and computational work [32-35] without blowing/suction, the pressure coefficient provided evidence of the separation bubble followed by re-attached flow, as observed in the oil flow pictures. A very slight increase and subsequent decrease in the experimental pressure curve is noticeable in the Figure 5. The position along the chord of this pressure peak is $30 \%$, similar to the location identified by the oil flow visualization photographs. The discussion above is supported by the skin-friction coefficients, as shown in Figure 6. In Figure 6 and 7, the calculations done with the k- $\omega$ SST transition model with blowing and suction shows that the separation bubble is subsided and the pressure hump moves upstream. But, the calculations done with the $\mathrm{k}-\mathrm{k}_{\mathrm{L}}-\mathrm{\omega}$ transition model with blowing and suction is not subsided the separation bubble. Consequently, the suction is better than blowing about elimination of the laminar separation bubble.

Unfortunately, no experimental work with blowing/suction for this aerofoil case is available under the above Ma and Re number conditions. Therefore, it is not possible to quantify the amount of change after the blowing/suction. However, at least on the qualitative grounds, it may be said that the effects of blowing are similar to expected results in which the blowing energizes the flow and alleviates the extent of the laminar separation bubble. Therefore, the underlying blowing/suction control mechanism appears to be the suppression of the separation bubble and the reduction of the upper surface pressure coefficients to increase lift and decrease the drag.

Figures 8 and 9 show the lift/drag ratios without (base) and with the blowing/suction with different jet angles, velocity ratios and locations using both transition models. It is noteworthy that the smallest blowing results are better larger blowing velocity ratios independent of the blowing locations while the largest suction results are better smaller suction velocity ratios independent of the suction jet locations. Yet, these results are qualitatively quite consistent with some earlier numerical predictions [23].

Figure 10 depicts the streamlines, intermittency and velocity profiles over the NACA 2415 aerofoil with and without blowing/suction at $\alpha=8^{\circ}$ using the $k$ - $\omega$ SST transition model. The effect of blowing/suction on the boundary layer transition point is displayed in which the k- $\omega$ SST transition model intermittency factor of $\gamma$ is used as an indicator. The intermittency factor of $\gamma$ having values less than one approximately indicates the extent of the laminar separation bubble. As shown in the figure, blowing/suction helps reduce the laminar flow region (on the right) as opposed to without blowing/suction (on the left). It appears that the onset of transition may have been pushed by $3 \%$ chord in the upstream direction from an approximate location of $29 \%$ (without blowing/suction, on the left) to $26 \%$ (with blowing/suction, on the right). The boundary layer velocity profiles in the same locations support the above conclusions that the blowing/suction suppresses the laminar separation bubble and help develop fuller boundary layer profiles. However, more attention is necessary here in order not to fall into self-deception because of the fact that $26 \%$ chord location is exactly where the jet blowing/suction occurs. This may very well be triggering the transition rather than altering the natural transition process. 


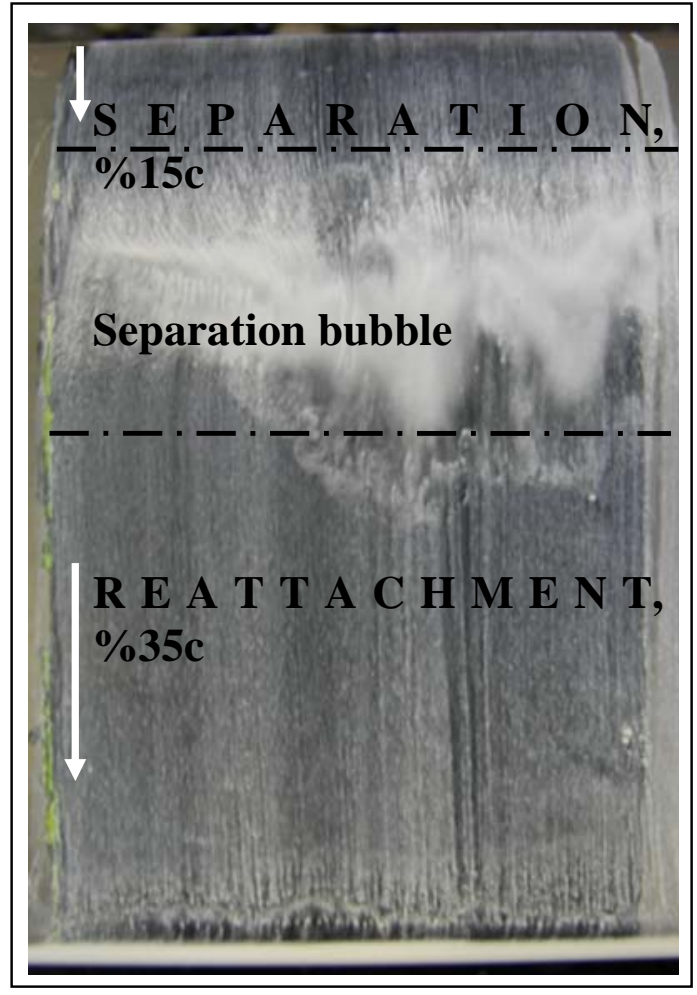

Figure 5. Oil flow visualization over the

NACA 2415 aerofoil at $\alpha=8^{\circ}$.

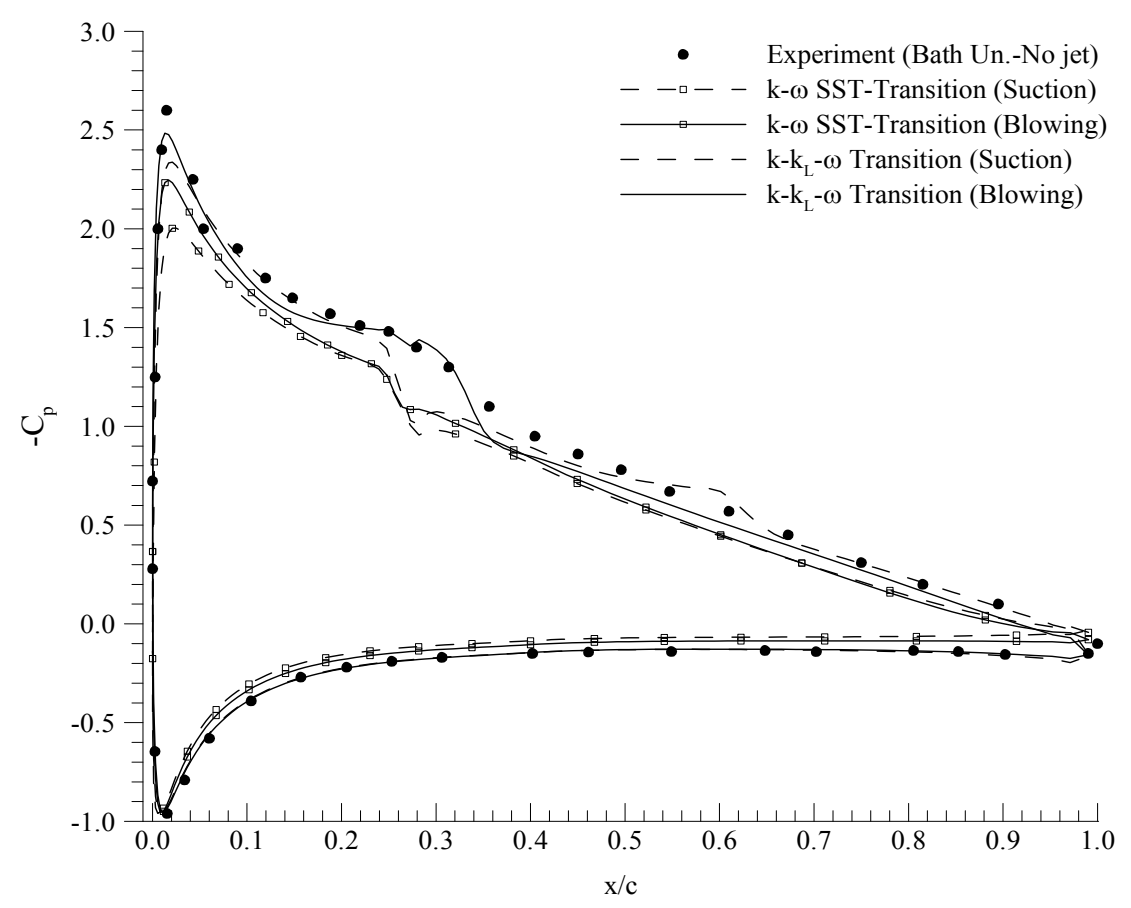

Figure 6. Comparison of numerical (Blowing; $\theta_{\text {jet }}=30^{\circ}, \mathrm{R}_{\mathrm{jet}}=0.003, \mathrm{~L}_{\mathrm{jet}}=\% 26 \mathrm{c}$, Suction; $\theta_{\text {jet }}=-30^{\circ}, \mathrm{R}_{\text {jet }}=0.156, \mathrm{~L}_{\mathrm{jet}}=\% 26 \mathrm{c}$ ) and experimental $\mathrm{C}_{\mathrm{p}}$ for the NACA 2415 aerofoil without and with blowing/suction at $\alpha=8^{\circ}$. 


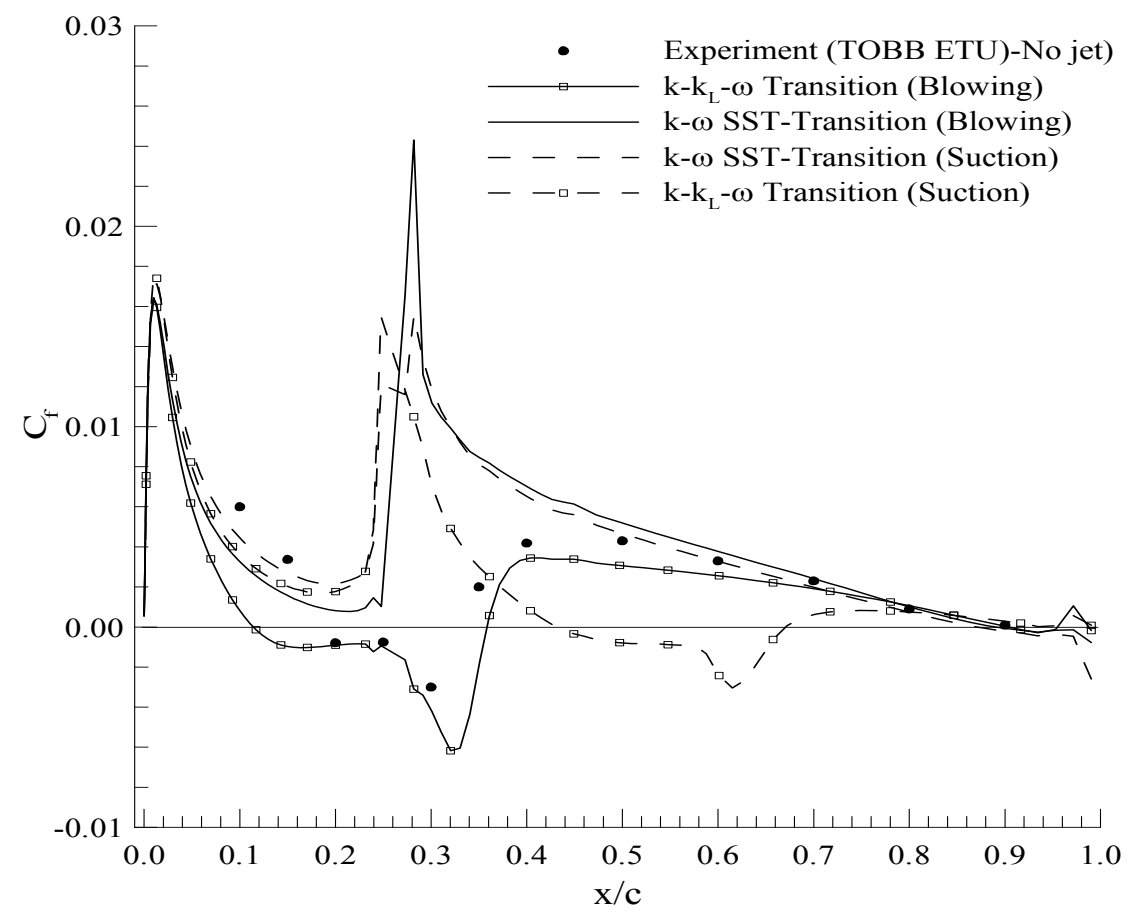

Figure 7. Comparison of numerical (Blowing; $\theta_{\mathrm{jet}}=30^{\circ}, \mathrm{R}_{\mathrm{jet}}=0.003, \mathrm{~L}_{\mathrm{jet}}=\% 26 \mathrm{c}$, Suction; $\theta_{\text {jet }}=-30^{\circ}, \mathrm{R}_{\mathrm{jet}}=0.156, \mathrm{~L}_{\mathrm{jet}}=\% 26 \mathrm{c}$ ) and experimental $\mathrm{C}_{\mathrm{f}}$ for the NACA 2415 aerofoil without and with blowing/suction at $\alpha=8^{\circ}$.

\section{Conclusion}

Low Reynolds number transitional flow over a NACA 2415 aerofoil using blowing/suction has been investigated at $\mathrm{Re}=2 \times 10^{5}$. Oil flow visualization experiments revealed that the flow over the single NACA2415 aerofoil is characterized by a laminar separation bubble on the suction surface at approximately $30 \%$ chord. Numerical simulations, employed by the FLUENT, showed that the recently developed $k-k_{L^{-}} \omega$ and the $k$ - $\omega$ SST transition models accurately predicted the location and extent of the separation bubble. The numerical and experimental using hot-wire anemometer in TOBB ETU velocity profiles over the upper surface of the NACA 2415 aerofoil at the angle of attack of $8^{\circ}$ show clearly that the separation bubble and the adverse velocity profiles at various chordwise positions. Both transition models and the $k-\omega$ SST turbulence model predict more pronounced reverse flow regions, but the $k-\varepsilon$ RNG turbulence model misses the separation bubble. Unfortunately, no experimental work with blowing/suction for this aerofoil case is available under the above Ma and Re number conditions. Therefore, it is not possible to quantify the amount of change after the blowing/suction. In the computational results, the blowing/suction control mechanism appears to be the suppression of the separation bubble and the reduction of the upper surface pressure coefficients to increase lift and decrease the drag. Furthermore, the smallest blowing results are better larger blowing velocity ratios independent of the blowing locations while the largest suction results are better smaller suction velocity ratios independent of the suction jet locations. Yet, these results are qualitatively quite consistent with some earlier numerical predictions [23]. 


\section{Acknowledgments}

M.Serdar GENÇ thanks the Foundation of Erciyes University for funding of visiting to North Dakota State University as academic visitor. Moreover, the authors thank the Scientific Research Projects Unit of Erciyes University for scientific research fund under the contract no: FBD-08-400, and Dr. Y. Bora SUZEN for helping about modelling of blowing/suction.

\section{References}

[1] Dannenberg, R. E., and Weiberg, J. A., "Section Characteristics of a 10.5-Percent Thick Airfoil with Area Suction as Affected by Chordwise Distribution of Permeability," NASA TN 2847, Dec. 1952.

[2] Stratford, B.S., "The prediction of separation of the turbulent boundary layer" Journal of Fluid Mechanics, 1959, Vol. 15, pp. 1-16.

[3] Schlichting, H. "Boundary Layer Theory" Sixth Edition. New York: Mcgraw-Hill, Inc, 1968.

[4] Liebeck, R.H. and Ormsbee, A.I., "Optimization of aerofoils for maximum lift” Journal of Aircraft, 1970, Vol. 7, No. 9-10, pp. 409-415.

[5] Bingham, G.J. and Chen, A.W, "Low-speed aerodynamic characteristics of an aerofoil optimized for maximum lift coefficient" NASA TN D-7071, 1972.

[6] Cebeci, T., Mosinskis, G.J. and Smith A.M.O, "Calculation of separation points in incompressible turbulent flows", Journal of Aircraft, 1972, Vol. 9, No. 9, pp. 618-624.

[7] Liebeck, R.H., "A class of aerofoils designed for high lift in incompressible flow", Journal of Aircraft, 1973, Vol. 10, No. 10, pp. 610-617.

[8] Abu-Ghannam, B.J. and Shaw, R., "Natural transition of boundary layers-the effect of turbulence, pressure gradient and flow history" Journal of Mechanical Engineering Science, 1980, Vol. 22, No. 5, pp. 213-228.

[9] Drela, M. and Giles, M.B., "Viscous-inviscid analysis of transonic and low Reynolds number aerofoils" AIAA Journal, 1987, Vol. 25, No. 10, pp. 1347-1355.

[10] Anderson, J.D. Jr., "Fundamentals of Aerodynamics" Second Edition, New York: Mcgraw-Hill, Inc, 1991.

[11] Choudhury, D., "Introduction to the renormalization group method and turbulence modeling” Fluent Inc. Technical Memorandum TM-107, 1993.

[12] Savill, A.M. "Some recent progress in turbulence modeling of by-pass transition", NearWall Turbulent Flows, Elsevier, 829, 1993.

[13] Wilcox, D.C., "Simulation of transition with a two-equation turbulence model" AIAA Journal, 1994, Vol. 32, No. 2, pp. 247-255.

[14] McCormick, B.W., "Aerodynamics, Aeronautics and Flight Mechanics” Second Edition, New York: John Wiley and Sons Inc., 1995.

[15] Wilcox, D.C., “Turbulence Modeling For CFD”, Second Edition, DCW Industries, 1998.

[16] Jie-Zhi Wu, Xi-Yun Lu, Andrew G. Denny, Meng Fan and Jain-Ming Wu, "Poststall Flow Control On An Airfoil By Local Unsteady Forcing”, Journal of Fluid Mechanics, Vol. 371, 1998, pp. 21-58.

[17] Catalin Nae, "Synthetic Jets Influence on NACA 0012 Airfoil at High, Angles of Attack", AIAA Atmospheric Flight Mechanics Conference and Exhibit, Boston, Massachusetts, August 10-12, 1998.

[18] A. Hassan, and R. D. Janakiram, "Effects of Zero-Mass Synthetic Jets on the Aerodynamics of the NACA 0012 Airfoil", Journal of the American Helicopter Society, Vol. 43, No. 4, Oct, 1998.

[19] Suzen, Y. B. and Huang, P.G., "Modeling of flow transition using an intermittency transport equation" ASME Journal of Fluid Engineering, 2000, Vol. 122, pp. 273-284. 
[20] Suzen, Y. B. and Huang, P.G., "Predictions of separated and transitional boundary layers under low-pressure turbine airfoil conditions using an intermittency transport equation" ASME Journal of Turbomachinery, 2003, Vol. 125, pp. 455-464.

[21] Lodefier, K., Merci, B., De Langhe, C. Dick, E., "Transition modeling with the SST turbulence model and intermittency transport equation" ASME Turbo Expo, Atlanta, GA, USA, June 16-19, 2003.

[22] Menter, F.R., Langtry, R.B., Likki, S.R., Suzen, Y.B., Huang, P.G. and Völker, S., "A correlation based transition model using local variables: Part I-model formulation", ASME-GT2004-53452, Proceedings of ASME Turbo Expo 2004, Vienna, Austria, pp. 57-67, 2004.

[23] Huang L., Huang P. G. and LeBeau R. P., Numerical Study of Blowing and Suction Control Mechanism on NACA0012 Airfoil, Journal of Aircraft ,vol. 41, No. 1, JanuaryFebruary 2004.

[24] Langtry, R.B. and Menter, F.R. "Transition modeling for general CFD applications in aeronautics" AIAA Paper 2005-0522, 2005.

[25] Walters D.K. and Leylek, J. H., "Computational fluid dynamics study of wake induced transition on a compressor-like flat plate" Transactions of the ASME, 2005, Vol. 127, pp. $52-55$.

[26] Misaka, T. and Obayashi, S. “A correlation-based transition models to flows around wings" AIAA Paper 2006-918, 2006.

[27] Fluent Incorporated, Fluent (V 6.4) and Gambit (V 2.1.6) User's Guides, 2007.

[28] Cutrone, L. De Palma, P., Pascazio, G. and Napolitano, M., "Predicting transition in twoand three-dimensional separated flows", International Journal of Heat Fluid Flow, 2008, Vol. 29, pp. 504-526.

[29] Kaynak, Ü. and Gürdamar, E., "Boundary-layer transition under the effect of compressibility for the correlation based model", AIAA-2008-0774, 46th AIAA Aerospace Sciences Meeting, Jan. 07-10 2008, Reno, Nv, USA.

[30] Shan H., Jiang L., Liu C., Love M. and Maines B., Numerical study of passive and active flow separation control over a NACA0012 airfoil, Computers \& Fluids, vol. 37, pp. 975992, 2008.

[31] Suluksna, K. and Juntasaro, E.. "Assessment of inmittency transport equations for modeling" International Journal of Heat Fluid Flow, 2008, Vol. 29, pp. 48-61.

[32] Genç M.S., Lock G. and Kaynak U., An Experimental and Computational Study of Low Re Number Transitional Flows over an Aerofoil with Leading Edge Slat, AIAA-20088877, ICAS 2008 Congress including the 8th AIAA 2008 ATIO Conference, Anchorage, Alaska, USA, 14-19 September 2008.

[33] Genç, M.S., Kaynak, Ü., Lock, G., Flow over an aerofoil without and with leading edge slat at a transitional Reynolds number, Pro.Ins Mech.Eng, Part G-Journal of Aerospace Engineering, in press.

[34] Genç, M.S., Kaynak, Ü., Kanat profili etrafındaki düşük Reynolds sayılı akım kontrolü ve türbülansa geçiş modellerinin etkisi, (in Turkish), ANOVA Kullanıcılar Konferansı-II, Ankara, Türkiye, 7-9 Ekim 2008.

[35] Genç, M.S., Kaynak, Ü., Lock, G., Hücum kenarı slatlı kanat profili etrafındaki düşük Re sayılı geçiş akışında akım ayrılmasının kontrolü, (in Turkish), II. Ulusal Havacılık ve Uzay Konferans1, UHUK 2008, İTÜ, İstanbul, Türkiye, 15-17 Ekim 2008.

[36] Genç, M.S., Kanat Profilleri Etrafındaki Düşük Reynolds Sayılı Akışın Kontrolü ve Aerodinamik Performansın İncelenmesi, (in Turkish), Doktora Tezi, Fen Bilimleri Enstitüsü, Erciyes Üniversitesi, Kayseri, Türkiye, 2009. 
Paper: ASAT-13-AE-11

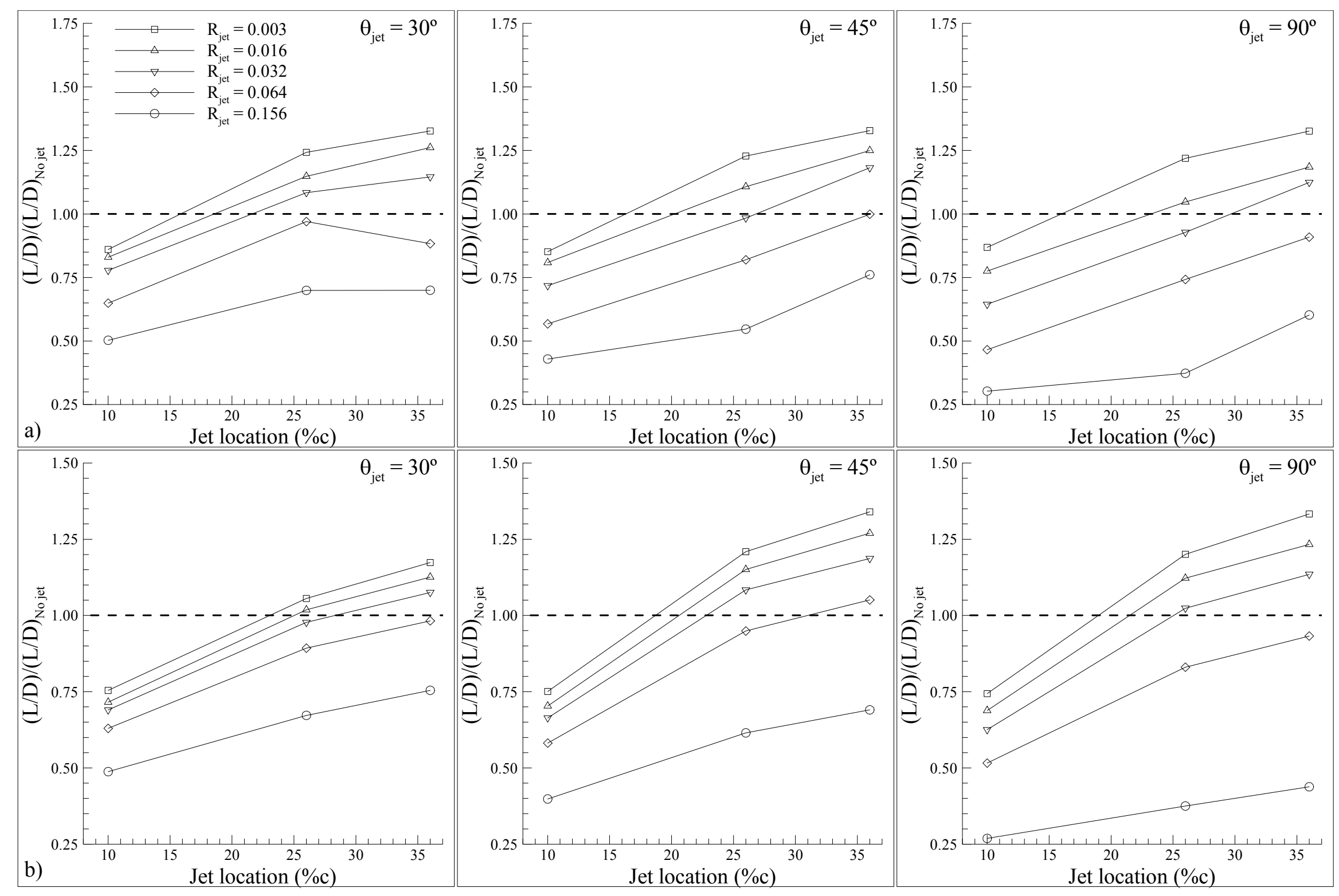

Figure 8. $\mathrm{L} / \mathrm{D}$ ratios of NACA2415 aerofoil at $\alpha=8^{\circ}$ with blowing a) the $\mathrm{k}-\mathrm{k}_{\mathrm{L}}-\omega$ transition model $\mathrm{b}$ ) the $\mathrm{k}-\omega \mathrm{SST}$ transition model. 
Paper: ASAT-13-AE-11

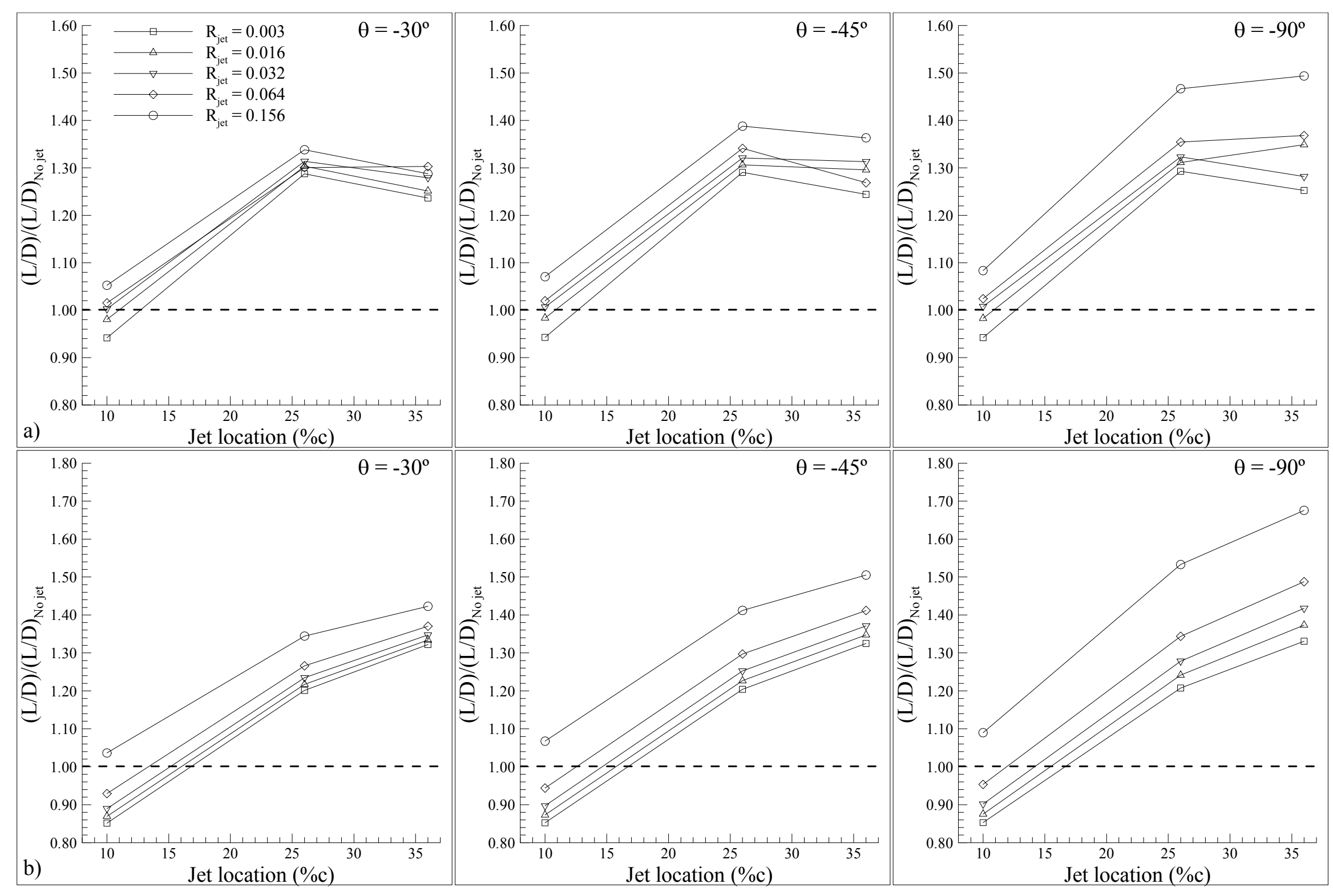

Figure 9. L/D ratios of NACA2415 aerofoil at $\alpha=8^{\circ}$ with suction a) the $k-k_{L}-\omega$ transition model b) the k- $\omega$ SST transition model. 
Paper: ASAT-13-AE-11

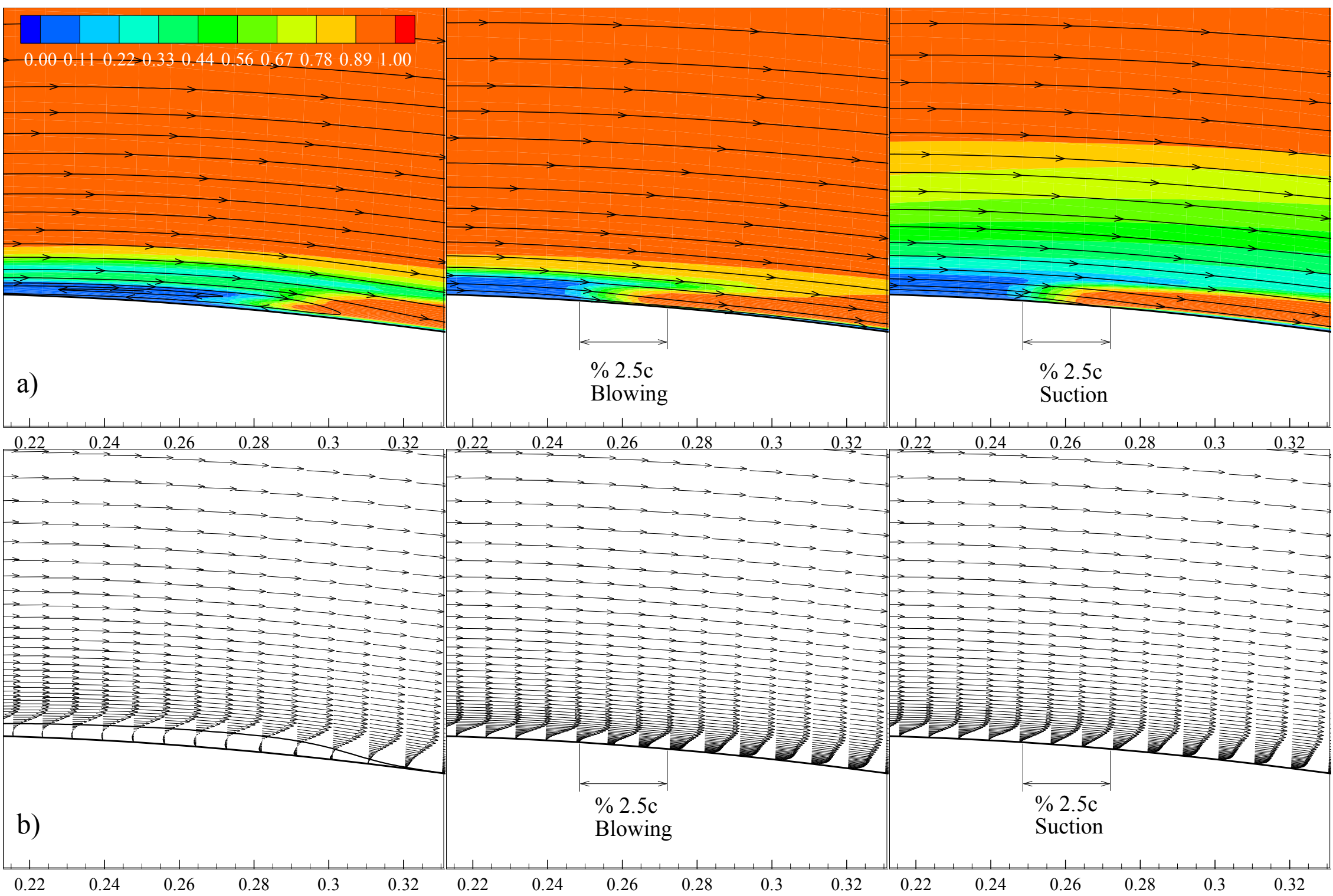

Figure 10. Distrubutions of a) the streamlines and intermittency $b$ ) the velocity profiles of NACA2415 aerofoil at $\alpha=8^{\circ}$ without and with suction $\left(\theta_{\mathrm{jet}}=-30^{\circ}, \mathrm{R}_{\mathrm{jet}}=0.032, \mathrm{~L}_{\mathrm{jet}}=\% 26 \mathrm{c}\right)$ and blowing $\left(\theta_{\mathrm{jet}}=30^{\circ}, \mathrm{R}_{\mathrm{jet}}=0.003, \mathrm{~L}_{\mathrm{jet}}=\% 26 \mathrm{c}\right)$ using the $\mathrm{k}-\omega \mathrm{SST}$ transition model 